\title{
Quantifying the effect of magnetopause shadowing on electron radiation belt dropouts
}

\author{
Y. Yu, J. Koller, and S. K. Morley \\ Space Science and Application, Los Alamos National Laboratory, Los Alamos, NM, USA \\ Correspondence to: Y. Yu (yiqun@lanl.gov)
}

Received: 19 August 2013 - Revised: 17 October 2013 - Accepted: 18 October 2013 - Published: 15 November 2013

\begin{abstract}
Energetic radiation belt electron fluxes can undergo sudden dropouts in response to different solar wind drivers. Many physical processes contribute to the electron flux dropout, but their respective roles in the net electron depletion remain a fundamental puzzle. Some previous studies have qualitatively examined the importance of magnetopause shadowing in the sudden dropouts either from observations or from simulations. While it is difficult to directly measure the electron flux loss into the solar wind, radial diffusion codes with a fixed boundary location (commonly utilized in the literature) are not able to explicitly account for magnetopause shadowing. The exact percentage of its contribution has therefore not yet been resolved. To overcome these limitations and to determine the exact contribution in percentage, we carry out radial diffusion simulations with the magnetopause shadowing effect explicitly accounted for during a superposed solar wind stream interface passage, and quantify the relative contribution of the magnetopause shadowing coupled with outward radial diffusion by comparing with GPS-observed total flux dropout. Results indicate that during high-speed solar wind stream events, which are typically preceded by enhanced dynamic pressure and hence a compressed magnetosphere, magnetopause shadowing coupled with the outward radial diffusion can explain about 60$99 \%$ of the main-phase radiation belt electron depletion near the geosynchronous orbit. While the outer region $\left(L^{*}>5\right)$ can nearly be explained by the above coupled mechanism, additional loss mechanisms are needed to fully explain the energetic electron loss for the inner region $\left(L^{*} \leq 5\right)$. While this conclusion confirms earlier studies, our quantification study demonstrates its relative importance with respect to other mechanisms at different locations.
\end{abstract}

Keywords. Magnetospheric physics (Solar windmagnetosphere interactions)

\section{Introduction}

Reductions of energetic electron flux in the outer radiation belt can generally be attributed to (a) adiabatic motion (i.e., Dst effect) (Kim and Chan, 1997) that radially transports particles adiabatically following a configuration change in the magnetosphere to conserve the three adiabatic invariants $(\mu$, $K, \phi$ ) and (b) nonadiabatic processes, such as the loss caused by pitch-angle scattering via various cyclotron wave-particle interaction, which leads to electron precipitation to the lowaltitude atmosphere (e.g., Lyons et al., 1972; Thorne et al., 2005; Summers et al., 2007a, b; Millan et al., 2007) as well as the loss across the magnetopause (i.e., magnetopause shadowing) into the interplanetary space (e.g., Desorgher et al., 2000; Ohtani et al., 2009; Ukhorskiy et al., 2006, 2011). Magnetopause shadowing is often caused by either an inward motion of the magnetopause that opens up the previously closed particle drift shells and depletes the particles or by outward motion of the particles that subsequently encounter the magnetopause boundary. Magnetopause shadowing is usually coupled with outward radial diffusion (Schulz and Lanzerotti, 1974) as the sudden loss to the magnetopause generates a sharp gradient that further drives particles outwards and then through the magnetopause onto open drift shells. While adiabatic processes allow electron flux in the storm recovery phase, depleted in the main phase, to return to its pre-storm level, many events associated with storm main-phase dropouts do not recover (Reeves et al., 2003). A statistical study by Li et al. (2009) found that the development of a storm leads to a net decrease of relativistic electrons in the outer radiation belt. The flux dropout in such a case can only be a result of nonadiabatic processes that permanently remove the energetic electrons from the system. Several different mechanisms fall into this "nonadiabatic" 
category; however, they are likely to act together and the relative role of each mechanism remains an open question (see Turner et al., 2013, for a review).

Observational analysis, aided by a variety of spacecraft measurements, provides a useful means of investigating the mechanism(s) possibly responsible for the rapid dropout in the outer radiation belt. For example, analysis of a number of satellite measurements (e.g., Bortnik et al., 2006; Millan et al., 2010; Turner et al., 2012) has suggested that loss to the magnetopause plays a major role in depleting the energetic electrons near and outside the geosynchronous orbit during storm main phases, while atmosphere precipitation has a much less significant contribution. Morley et al. (2010b) studied a rapid loss of energetic electrons observed by the GPS constellation and found that the loss at and beyond geosynchronous orbit is highly correlated with the motion of the magnetopause. Matsumura et al. (2011) presented the correlation between the outer radiation belt boundary location and the magnetopause location, suggesting that the magnetopause shadowing plays a major role in the variation of the outer radiation belt. Besides the above case studies, Meredith et al. (2011) conducted a superposed epoch analysis of energetic electron dropouts during 42 high-speed solarwind-stream- (HSS) driven storms and found no evidence for enhanced precipitation of $\mathrm{MeV}$ electron during the mainphase dropout and suggested that the decrease in the $\mathrm{MeV}$ electron flux is not caused by the precipitation to the atmosphere. This result was confirmed using a different set of HSS drivers by Hendry et al. (2012).

Some of the above studies qualitatively compared the precipitation signature and the dropout in the electron radiation belt, and since no significant precipitation was observed in the high $L$ shell, the authors concluded that the magnetopause shadowing contributes most of the electron dropout. Some of the studies correlated the radiation belt variability with the magnetopause location and suggested the similar conclusion based on a high-correlation relationship. None of these studies, however, directly examined the loss to the solar wind since the loss out of the magnetopause boundary is difficult to measure.

On the other hand, radiation belt numerical modeling (e.g., Desorgher et al., 2000; Brautigam and Albert, 2000; Kim et al., 2008, 2010, 2012; Su et al., 2011b; Subbotin et al., 2011) has the advantage for researchers that by manipulating the code and switching on/off the mechanisms of interest, their relative importance in the flux dropout can be revealed. For example, Kim et al. (2008) numerically studied the extent of the drift loss/magnetopause shadowing under different solar wind pressure or IMF $B z$ conditions through drift path tracing, using the guiding-center method, and estimated the relative decrease of the $\mathrm{MeV}$ electron flux when pressure or IMF $B z$ is enhanced. Using a variable boundary condition from the satellite CRRES, Shprits et al. (2006) simulated the main-phase depletion and concluded that the radial diffusion can effectively propagate the nonadiabatic boundary flux variations down to $L^{*}=4$ and that the possible magnetopause loss in the variable boundary conditions together with the radial transport can account for the mainphase flux dropout. Su et al. (2011b) investigated the relative contribution of different loss mechanisms by comparing a CRRES-observed dropout with 3-D electron radiation belt modeling by gradually including more processes in the code. They found that the magnetopause shadowing together with adiabatic transport overestimate the electron flux outside $L$ of 5 but underestimate that inside $L$ of 5 and that a further introduction of the plume wave-particle interaction remarkably captures the $1 \mathrm{MeV}$ electron flux profile.

These modeling studies shed some light on the relative importance of magnetopause shadowing in depleting the electron radiation belt, but again, none of these simulations quantitatively reported the contribution of magnetopause shadowing. Exactly how much of the loss is caused by this mechanism? What is the relative percentage of its contribution in the total dropout? This study will tackle this issue by using GPS observations combined with radial diffusion simulations to quantify its contribution in HSS ensemble events.

In radiation belt modeling, the outer boundary, with appropriate boundary conditions, can highly control the dynamic evolution of the radiation belt. The outer boundary condition can propagate any loss or acceleration information inward, influencing the dynamics in the radiation belt including an ultimate depletion of the electron population away from the radiation belt (e.g., Shprits et al., 2006). The outer boundary of the radiation belt is commonly placed at a fixed drift shell to model the trapped radiation belt electron population, with a boundary condition derived from measurements such as geosynchronous observations or CRESS and POLAR measurements (e.g., Brautigam and Albert, 2000; Shprits et al., 2006) or based on a kappa-type distribution function as used in Su et al. (2011a).

Such a fixed drift shell is incapable of representing the time-varying magnetopause boundary, and the data-inferred boundary condition carries the net outcome of competing mechanisms. Therefore the real loss into the solar wind cannot be truly identified. To study the effect of magnetopause shadowing on the depletion of energetic electrons, the boundary of the radiation belt modeling is required to be explicitly set at the last closed drift shell across which the ultimate electron loss occurs. This last closed drift shell, however, is not only time varying but also $K$ (bounce-motion-related adiabatic invariant) dependent, which is associated with the drift shell splitting in a nondipolar magnetic field (Roederer, 1970). In this study, a $K$-dependent boundary is implemented in the radial diffusion model to be consistent with the simulation of the trapped electrons under a particular $K$ coordinate. This boundary also varies with time according to the upstream solar wind conditions. Such a boundary can account for the magnetopause shadowing as well as the drift splitting, and the amount of electron loss into the solar wind can be explicitly quantified. 
This paper primarily focuses on quantifying the relative contribution of magnetopause shadowing in the electron flux dropouts during 67 HSS events (from Morley et al., 2010a) by simulating the magnetopause shadowing effect in the 1$\mathrm{D}$ radial diffusion model. Section 2 describes the GPS data that will be used to obtain the total electron loss during HSS dropout events. Section 3 presents the methodology for carrying out radial diffusion simulations using a time-varying, $K$-dependent last closed drift shell boundary to explicitly capture the magnetopause shadowing. The quantitative comparison with GPS observations of the relative flux decrease during the superposed HSS event is shown in Sect. 4.

\section{Data}

In this study, we use the superposed epoch results of interplanetary and geomagnetic parameters from 67 HSS events spanning from year 2005 to 2008 studied by Morley et al. (2010a) (Fig. 1). The zero epoch time is taken at the time when the east-west solar wind flow $\left(V_{y}\right)$ deflection reverses. The median Dst index minimizes around $-22 \mathrm{nT}$, and the median $\mathrm{Kp}$ approaches 4 at maximum. Although over $75 \%$ of these events have a minimum Dst above $-35 \mathrm{nT}$, and would therefore typically not be identified as storms (Loewe and Prölss, 1997), they display a consistent response that is qualitatively storm-like. In the following, we will refer to the superposed median parameters of these events as a "typical HSS event". The rapid increase in the solar wind speed and solar wind number density in these events leads to enhancement of the solar wind dynamic pressure. This ensures an inward motion of the magnetopause and thereby a compressed magnetosphere during the HSS event, providing a good example of examining the real electron loss due to magnetopause shadowing. Note that the solar wind at epoch of 0 is already in the transition to a high dynamic pressure, so the last closed drift shell (shown later) starts to move inward before zero epoch.

The GPS data used here are obtained from the CXD (combined X-ray dosimeter) instrument package (Distel et al., 1999). Energetic electrons are measured by two subsystems: the low-energy particle (LEP) subsystem resolves 0.14 to $>1.25 \mathrm{MeV}$ electrons into five energy channels; the highenergy X-ray and particle (HXP) subsystem resolves 1.3 to $>5.8 \mathrm{MeV}$ electrons into six energy channels (see also Denton and Cayton, 2011). The CXD electron count rates are inverted to obtain the omni-directional differential number flux, $j$, by solving the spectral inversion function (Ginet et al., 2013)

$\boldsymbol{y}=\delta t \int \boldsymbol{G}(E) j(E) d E+\boldsymbol{b}$,

where $\boldsymbol{y}$ is a vector of observed counts, $\delta t$ is the integration time, $\boldsymbol{G}$ is a vector of energy-geometric factors (response functions), $j(E)$ is the omni-directional differential particle

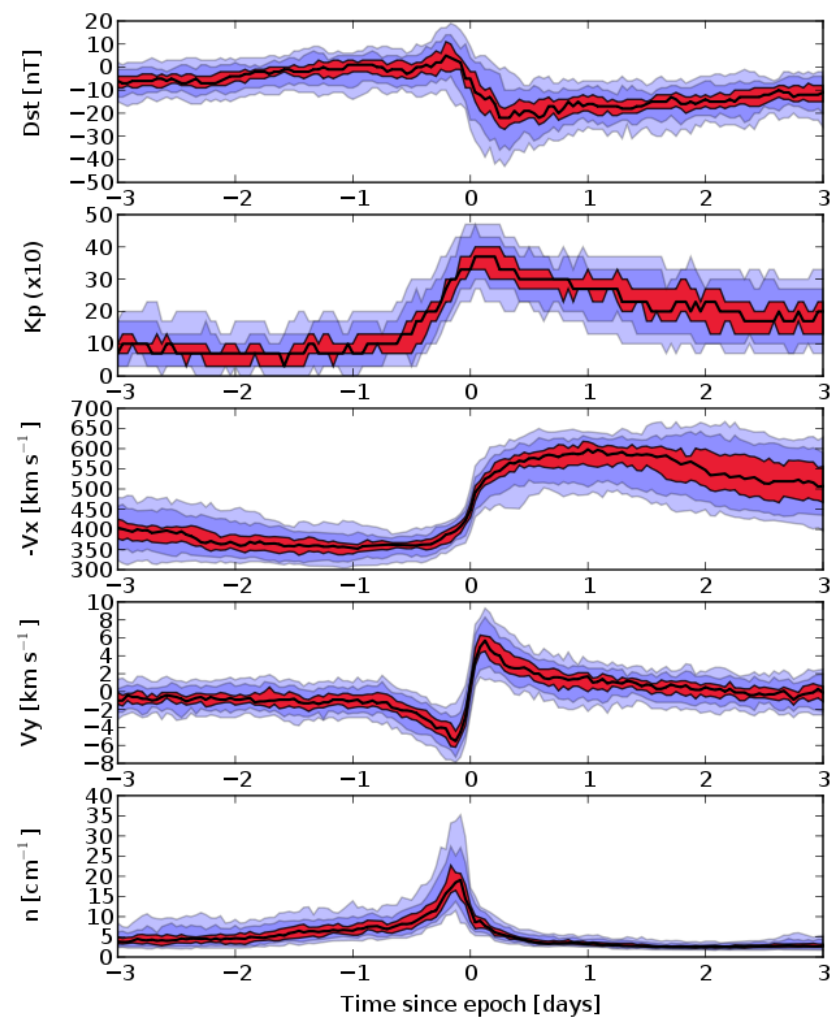

Fig. 1. Superposed solar wind and magnetospheric conditions of 67 high-speed solar-wind stream (HSS) events that occurred during the period 2005-2008, from Morley et al. (2010a). The zero epoch time is taken at the time when the east-west solar wind flow $\left(V_{y}\right)$ deflection reverses. The median Dst index minimized around $-22 \mathrm{nT}$ and the Kp approaches 4 at maximum. The increased solar wind velocity and density during the HSS events result in a compressed magnetosphere and thus a good example of studying the magnetopause shadowing.

flux at energy $E$, and $\boldsymbol{b}$ is a vector of expected background counts. The instrument response functions have been derived through extensive Monte Carlo modeling of the instrument package.

Following previous work, and consistent with observation (Cayton et al., 1989; Varotsou et al., 2008; Denton et al., 2010), a relativistic Maxwellian energy spectrum is assumed in the inversion procedure:

$j(E)=E\left(1+E / E_{0} / 2\right) e^{\left(q_{1}+q_{2} E\right)}$,

where the constant $E_{0}$ is the rest energy of the particle species (511 keV for electrons). The inversion of Eq. (1) is carried out using InvLib, a C inversion library developed by Paul O'Brien at Aerospace Corporation and used in the data preparation for the AE9/AP9/SPM radiation belt climatology model (see Ginet et al., 2013, and references therein). For each time we assess the goodness of fit of the relativistic Maxwellian and discard those data that cannot adequately be described by the chosen energy spectrum. 
In order to compare with the following simulation results, the GPS omni-directional differential flux is sorted in the drift shell $L^{*}$ coordinate, averaging over magnetic local time. The determination of the drift shell $L^{*}$ generally requires a global magnetospheric configuration as the computation involves numerical tracing of global magnetic field lines (Roederer, 1970). Instead, in this study, the recently developed $L^{*}$ neural network (Koller et al., 2009; Koller and Zaharia, 2011; Yu et al., 2012) is employed to compute the drift shells where the GPS instruments were located during all of the $67 \mathrm{HSS}$ events. The $L^{*}$ neural network has been proven to be efficient while preserving high accuracy with a prediction efficiency of $99.7 \%$ (Yu et al., 2012) when comparing to the traditional time-consuming numerical fieldline tracing method. The $L^{*}$ neural network employed here was trained from the T89 empirical magnetic field model (Tsyganenko, 1989) and was run through the SpacePy software package (Morley et al., 2010c).

\section{Methodology}

The one-dimensional radial diffusion model simplified from the Fokker-Plank equation with a loss term is used to simulate the evolution of phase space density (PSD) distribution $f\left(L^{*}, t\right)$ of the trapped radiation belt energetic electron during the above superposed HSS event:

$$
\left.\frac{\partial f\left(L^{*}, t\right)}{\partial t}\right|_{\mu, K}=L^{* 2} \frac{\partial}{\partial L^{*}}\left(\frac{D_{\mathrm{LL}}}{L^{* 2}} \frac{\partial f\left(L^{*}, t\right)}{\partial L^{*}}\right)-\frac{f\left(L^{*}, t\right)}{\tau},
$$

where $D_{\text {LL }}$ is the radial diffusion coefficient adapted from the empirical result in Brautigam and Albert (2000):

$D_{\mathrm{LL}}\left(\mathrm{Kp}, L^{*}\right)=10^{(0.506 \mathrm{Kp}-9.325)} L^{* 10}, \mathrm{Kp} \in[1,6]$,

where the Kp index follows the superposed median value of the above 67 HSS events. The electron lifetime $\tau$ is set to be one minute once it migrates across the magnetopause, implying a prompt loss of radiation belt electrons into the solar wind. Different timescales were tested, including 0.1, 5, and $10 \mathrm{~min}$, and no significant difference is found in the PSD at $L^{*} \leq 7.0$, indicating that the selected lifetime would not considerably influence the results near geosynchronous orbit as long as it is in timescales of drift periods.

\subsection{Initial condition and outer boundary}

The initial condition is determined from DREAM (the Dynamic Radiation Environment Assimilation Model) (Reeves et al., 2012) for different $(\mu, K)$ combinations. DREAM performs data assimilation using an ensemble Kalman filter technique (Koller et al., 2007) by combining PSD data with a 1-D radial diffusion model. The input PSD data are converted from flux observations from three LANL-GEO satellites, one GPS satellite (ns41), and the POLAR spacecraft in the second half of 2002. The conversion from flux to PSD

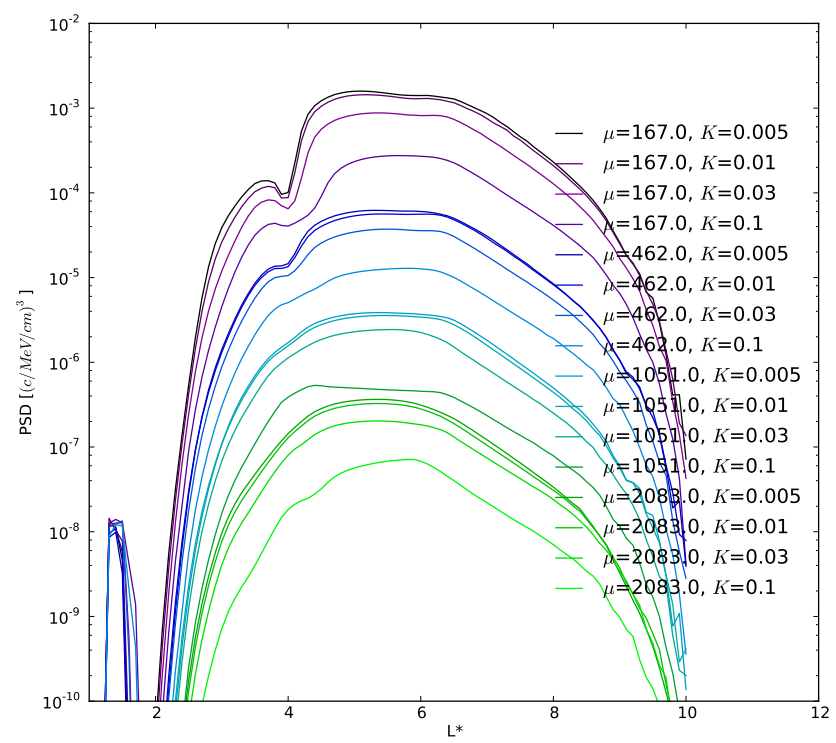

Fig. 2. The initial condition used in the radial diffusion simulations for different $\mu$ and $K$ combinations. The initial conditions are obtained from averaging over quiet-time radiation belt data assimilation results in 2002 after running DREAM (the Dynamic Radiation Environment Assimilation Model; Reeves et al., 2012) using data from three LANL-GEO, one GPS, and the POLAR spacecraft.

follows the algorithms described in Chen et al. (2005). The PSD is assimilated at different $\mu$ and $K$ coordinates, i.e., $\mu$ at $167.0,462.0,1051.0$, or $2083.0 \mathrm{MeV} \mathrm{G}^{-1}$ and $K$ at 0.005 , $0.01,0.03$, or $0.1 \mathrm{G}^{1 / 2} R_{E}$. The quiet-time- (Dst $>-20 \mathrm{nT}$ ) assimilated PSD $\left(\mu, K, L^{*}\right)$ during this half-year period is then averaged over time, which is subsequently applied as the initial condition in this study as displayed in Fig. 2.

The outer boundary is a crucial element in the radiation belt modeling owing to its high-impact modulation on the systematic variation. In this study, the outer boundary in the radial diffusion model represents the last closed drift shell, which is the magnetopause boundary. We will conduct a group of radial diffusion simulations with different $K$ values, so the outer boundary (i.e., the last closed drift shell) must be $K$ consistent, i.e., the last closed drift shell is a function of $K$ value. Because of the drift shell splitting (Roederer, 1970) and ever-changing magnetospheric configurations, the $K$ parameter cannot be simply related to one pitch angle on the last closed drift shell. Therefore the following steps are carried out to obtain the outer boundary at a certain $K$ parameter $\left(K_{0}\right)$ : (1) a bisection iterative method is applied in tracing each drift shells from the midnight meridian until the last closed drift shell $L_{\max }^{*}(\alpha)$ is determined for a set of equatorial pitch angle $\alpha$ ranging from 20 to $90^{\circ}$; (2) the above procedure also provides the corresponding $K$ parameter at the last closed drift shell for a specific equatorial pitch angle, i.e., $K(\alpha)$; (3) these $L_{\max }^{*}(K(\alpha))$ are then interpolated into $L_{\max }^{*}\left(K_{0}\right)$. 

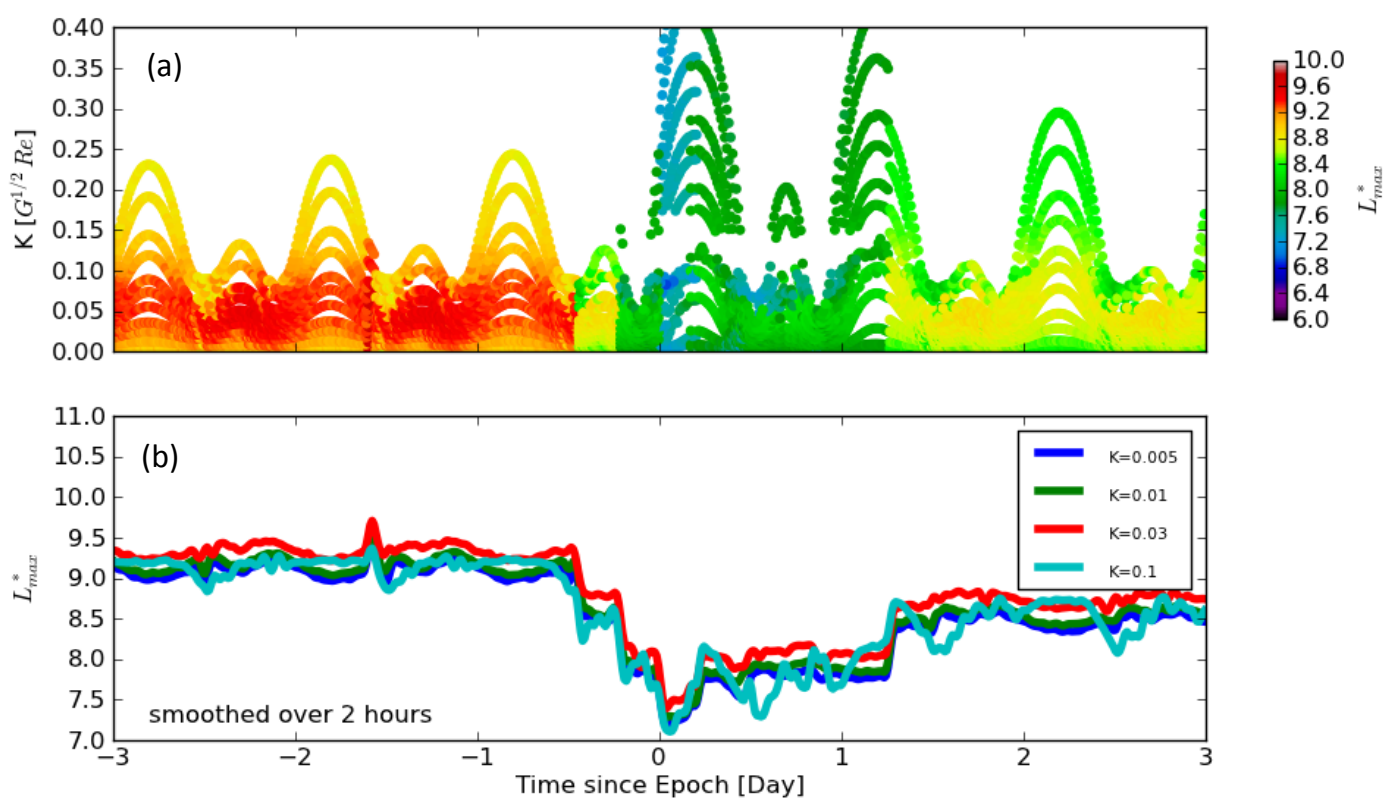

Fig. 3. (a) The color contour of the last closed drift shell $\left(L_{\max }^{*}\right)$ as a function of $K$ parameter ( $y$ axis). The $L_{\max }^{*}$ is obtained by numerically tracing magnetic field lines with the bisection technique on the midnight equator with a particular pitch angle using the T89 magnetic field model. Different pitch angles spanning from 20 (top trace) to $90^{\circ}$ (bottom trace) are used to calculate the last closed drift shell $L_{\text {max }}^{*}$ and corresponding $K$ parameter. The traces represent the result of different pitch angles. (b) The interpolated last closed drift shell for specific $K$ values. This represents the magnetopause location in the radiation belt radial diffusion model.

Figure 3a shows the last closed drift shell obtained from different pitch angles (shown by traces along the time, with the top ones computed with smaller pitch angles). The last closed drift shell appears to be more outward (darker red) when the pitch angle is intermediate (around $50^{\circ}$ ) than that with smaller pitch angle or larger pitch angle. The possible reason is that the particle with intermediate pitch angles undergoes a Shabansky orbit (Shabansky, 1971; Öztürk and Wolf, 2007; Ukhorskiy et al., 2011) in which it does not come across the equator but bounces within one hemisphere, allowing a larger drift shell until it encounters the magnetopause boundary (see Kim et al., 2008, for an illustration). The quasi-periodic daily evolution of the last drift shell is caused by the warping of the tail current sheet across the magnetic equator in the T89 magnetic field model (Tsyganenko, 1989) that is used to account for the geodipole tilt angle. During the storm main-phase of the superposed HSS event, the last closed drift shell clearly moves inwards (blue coloring) and the $K$ parameter increases because of the stretching in the magnetotail. Figure $3 \mathrm{~b}$ shows the interpolated last closed drift shell at particular $K$ values for the desired simulations. For smaller $K$ values (around 0.005 and $0.01 \mathrm{G}^{1 / 2} R_{E}$ ), the last closed drift shell shows a small discrepancy; however, it can significantly differ (up to 0.5 ) when $K$ becomes larger.

\subsection{From simulated PSD to flux}

Exhibited in Fig. 4a is the phase space density simulation result for $(\mu, K)$ of $\left(462.0 \mathrm{MeV} \mathrm{G}^{-1}, 0.03 \mathrm{G}^{1 / 2} R_{E}\right)$ with drift loss to the corresponding boundary. The phase space density is significantly reduced near the epoch time when the enhanced solar wind dynamic pressure compresses the magnetosphere, resulting in permanent electron loss to the solar wind. The goal of this work is to quantify the effect of magnetopause shadowing on the flux dropout of trapped radiation belt electrons via quantitative comparisons between simulation results and GPS observation. While GPS measures count rates, these are inverted to omni-directional differential flux as a function of energy (in Sect. 2); in order to allow for direct comparison with the GPS flux observation, the simulated PSD $f\left(\mu, K,{ }^{*}\right)$ results are also converted to omnidirectional differential flux $j(E)$. The conversion procedure is hereby summarized as follows:

1. "Fly" 24 stationary virtual satellites on the midnight equator from 4.0 to $10.0 R_{E}$ with $0.25 R_{E}$ separation and calculate $L^{*}(\alpha)$ at these positions with 18 different pitch angles (from 5 to $90^{\circ}$ ) using the T89 $L^{*}$ neural network technique (Yu et al., 2012) ( $K$ parameter is a by-product along this step).

2. Interpolate $L^{*}\left(\alpha\left(K_{0}\right)\right)$ into the drift shell with the $K$ value in the simulations (i.e., $L^{*}(K)$ ), and extract from the simulated PSD $f\left(\mu, K, L^{*}\right)$ for all virtual satellites, which together should reproduce the original simulated radiation belt PSD environment (Fig. 4b). 

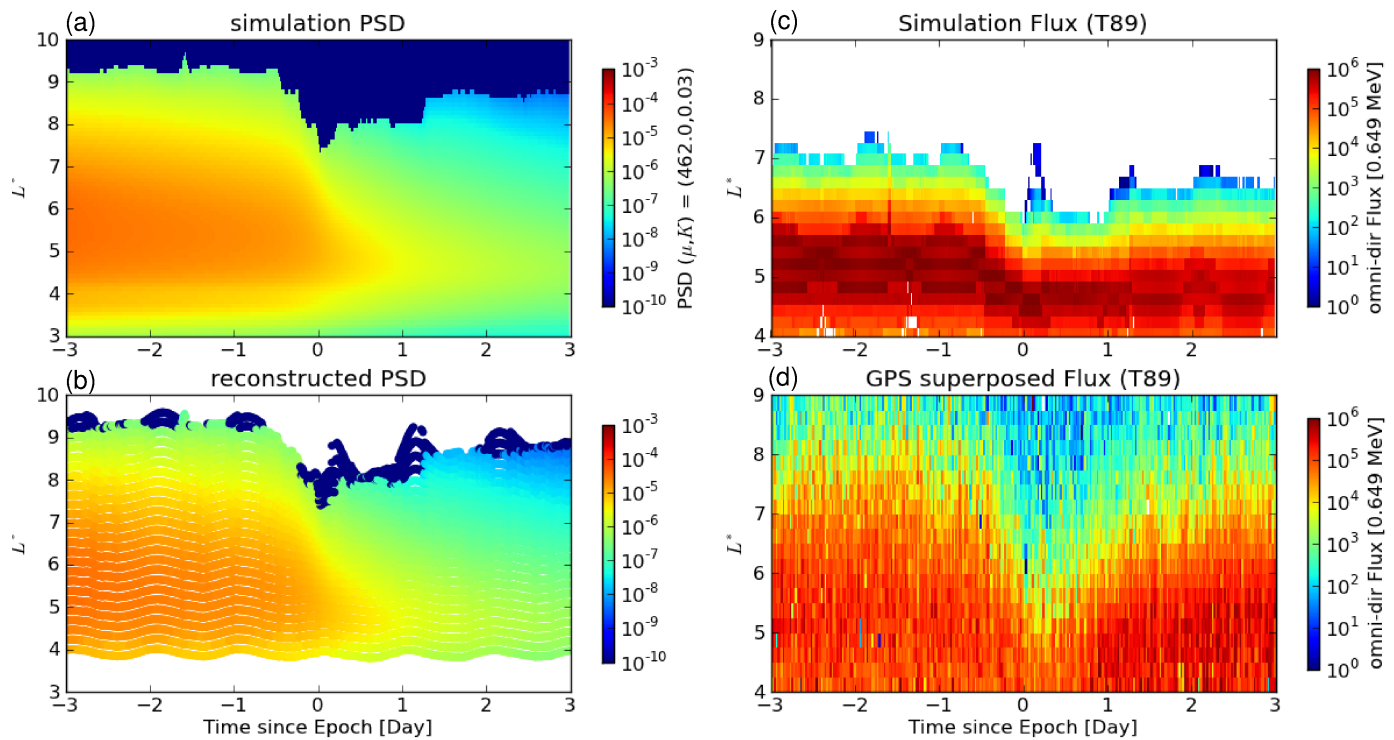

Fig. 4. (a) One example of the simulated phase space density at $\mu$ of $462.0 \mathrm{MeVG}^{-1}$ and $K$ of $0.03 G^{1 / 2} R_{E}$ in the superposed HSS event with the magnetopause shadowing explicitly accounted for. (b) The reconstructed PSD from 24 stationary "satellites" at the midnight equatorial plane. (c) The converted flux in $L^{*}$ space at energy level of $0.649 \mathrm{MeV}$ following the procedure described in Sect. 3.2. (d) The superposed GPS flux observations from $67 \mathrm{HSS}$ events at the energy level of $0.649 \mathrm{MeV}$.

3. The extracted PSD $f\left(\mu, K, L^{*}\right)$ at each virtual satellite is subsequently converted to flux $j(\alpha, E, r)$ using the following equation:

$$
\begin{aligned}
j(\alpha, E, r)= & f\left(\mu, K, L^{*}\right) \cdot\left(E^{2}+2 E_{0} E\right) / \\
& 3.3 \times 10^{-8}\left(\frac{c}{\mathrm{MeV} \mathrm{cm}}\right)^{3},
\end{aligned}
$$

where the differential flux $j$ is in units of $\mathrm{cm}^{-2} \mathrm{~s}^{-1} \mathrm{sr}^{-1} \mathrm{keV}^{-1}$ and PSD $f$ is in units of $c^{3} /(\mathrm{MeV} \mathrm{cm})^{3}$ (Chen et al., 2005). The differential flux is consequently integrated over the solid angle to obtain the omni-directional flux $j(E ; r)$.

4. Sort the flux output from all virtual satellites by $L^{*}$ (taking the average at the $L^{*}$ grid if overlap occurs for multiple satellite results), which is shown in Fig. 4c. The relative change in the omni-directional flux from pre-dropout to the minimum dropout time will be compared with the observed flux change as described below.

\section{Quantitative results}

Figure $4 \mathrm{c}$ shows the simulated omni-directional flux (converted from simulated phase space density) at $0.65 \mathrm{MeV}$ after carrying out the above procedures. The reason that it lacks data in larger drift shells is because the conversion from PSD to the flux invokes interpolation over a particular energy grid (here is $0.65 \mathrm{MeV}$ ) that falls outside the available energy range associated with the prescribed $(\mu, K)$ combinations and no extrapolation is done in this study for the purpose of preserving the overall accuracy. Figure $4 d$ displays the GPS-observed omni-directional flux superposed from 67 HSS events. Both the simulated and observed flux show a rapid decrease across a wide $L^{*}$ range within a few hours immediately after the magnetopause boundary moves inward. Although the magnitude of the simulated flux is higher than the observation within $L^{*}$ of 4.5-6.0, the simulation nearly captures the rapid flux decrease with the same timescale in the dropout time. Note that no source mechanism is introduced in the entire simulation, which may be sufficiently valid during the HSS dropout time period because generally no large competing acceleration (source) process takes place during the dropout time period (a similar assumption was made by Kim et al. (2010)). Such an exclusion of the source term in the model also explains why the observed flux returns to a higher level in the recovery phase than the simulated flux. Since the simulation reproduces the rapid flux dropout to a large degree despite the different magnitude in the flux than the observation, only the relative change in the dropout time (from the pre-dropout to the minimum dropout time) is examined in this study to investigate how much of the total radiation belt electron loss (as observed by the GPS) can be explained by drift loss to the magnetopause boundary coupled with outward radial diffusion (as implemented in the simulation). Figure 5 shows the flux at different $L^{*}$ locations from the simulation and GPS observations. We use the shaded regions spanning over the time axis to obtain the averaged flux for two "instances" (i.e., pre-dropout time and 
minimum dropout time). The relative flux change is parameterized by

$$
\frac{\left|j_{\min }-j_{\text {pre }}\right|}{j_{\text {pre }}}
$$

where "min" stands for the time of minimum flux dropout (i.e., $5 \mathrm{~h}$ after the epoch zero time, with $\pm 5 \mathrm{~h}$ spanning to average for better statistics), and "pre" means the pre-dropout time ( $12 \mathrm{~h}$ before the epoch, with $\pm 3 \mathrm{~h}$ spanning). By taking the ratio between the relative flux change in the simulation resulting from the magnetopause shadowing coupled with the outward radial diffusion, and the relative flux change in the observation resulting from all loss mechanisms, we can quantify the percentage contribution of the magnetopause shadowing plus the radial diffusion in depleting the electron flux during the dropout period.

Figure 6a shows the simulated radial profile of the flux at the pre-dropout and minimum dropout times. After the sudden dropout, the flux peak (at $L^{*} \approx 5$ ) shifts inward, diffusing in both directions. The inner region flux is enhanced with the inward diffused flux, while the outer region flux is significantly decreased. This decrease is a result of, as implemented in the radiation belt modeling, the drift loss to the open drift shell outside the magnetopause caused by the inward motion of the magnetopause and the outward radial diffusion that is further enhanced due to a sharp gradient at the magnetopause boundary.

Since the goal of this study is to quantify how much of the total loss in the radiation belt electrons is caused by the drift loss coupled with the outward radial diffusion, the relative reduction in the flux from pre-dropout to the minimum dropout time is calculated using Eq. (6) and depicted in Fig. 6b. Shown for comparison is the observed relative flux change during the same time period (dot line). The total electron flux observed by the GPS decreases by about 67 to $95 \%$ for $L^{*}$ of 5.0 to 6.0 during the dropout time, with a larger drop in the outer region. The simulation demonstrates that the flux decreases by about 40 to $90 \%$ in the same drift shell region, roughly following the same decreasing tendency while moving outward. This suggests that the loss mechanisms specified in the simulation, i.e., magnetopause shadowing plus outward radial diffusion, can approximately explain 60 to $99 \%$ of the sudden electron loss during the superposed HSS event near the geosynchronous orbit (Fig. 6c). Outside $L^{*}$ of 5.0, the above loss mechanism can explain more than $93 \%$ of the total dropout, but its contribution to the inner region $\left(L^{*} \leq 5\right)$ is much less $(60 \%)$.

\section{Discussion}

Note that by comparing the flux quantity in a certain energy level, the adiabatic effect cannot be completely neglected. It can lead to a change in electron population observed at a particular spatial position when the magnetosphere varies.
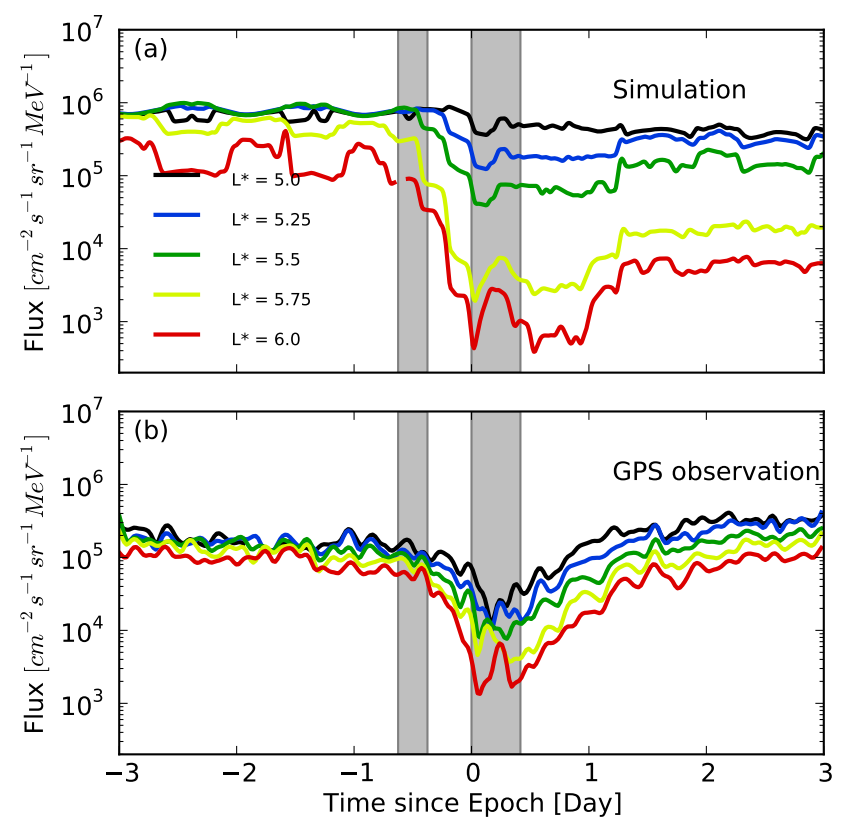

Fig. 5. The flux at different $L^{*}$ locations from (a) simulation and (b) GPS observations. The two shaded regions represent two "instances" in an average sense: pre-dropout time $(12 \mathrm{~h}$ before the epoch zero time, with $\pm 3 \mathrm{~h}$ spanning), and minimum dropout time ( $5 \mathrm{~h}$ after the epoch zero time with $\pm 5 \mathrm{~h}$ spanning), respectively.

Nevertheless, the phase space density in the adiabatic coordinate system in Fig. 4a clearly demonstrates the decrease of electron content in the outer radiation belt, indicating that the electrons are truly lost due to the drift loss and outward radial diffusion. In actual fact, the adiabatic effect contributing to the flux variation during the dropout event is potentially minimized by studying the flux in the drift shell $L^{*}$ coordinate. Furthermore, even if any adiabatic effect remains because of the changing magnetospheric configuration, it makes, however, the same contribution in both the observation and simulation since the same magnetospheric magnetic field model (T89) is employed. Therefore comparing the relative flux change will rule out the same adiabatic effect in both the simulation and observation.

Earlier observational works (Bortnik et al., 2006; Millan et al., 2010; Loto'aniu et al., 2010; Turner et al., 2012) conducted case studies of radiation belt dropouts during storm events and suggested that the combination of magnetopause shadowing and outward radial transport can explain the observed dropout near and outside the geosynchronous orbit. Numerical studies using a radial diffusion model with fixed boundary locations and time-dependent boundary conditions inferred from satellite measurements (e.g., Brautigam and Albert, 2000; Miyoshi et al., 2003; Shprits et al., 2006) were able to reproduce main-phase dropouts in the outer radiation belt energetic electron content. The study presented here, unlike the above case studies and simulations with fixed boundary locations, simulated an ensemble of HSS events 

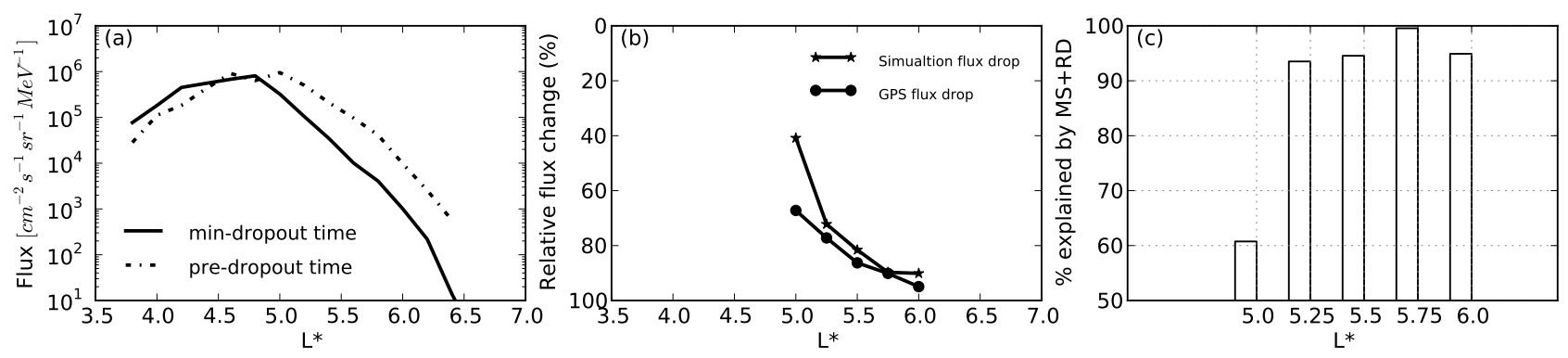

Fig. 6. (a) The radial profile of the $0.649 \mathrm{MeV}$ simulated flux at two "instances": pre-dropout and minimum dropout time. (b) The relative flux change in the simulation and in the GPS observation during the storm main phase (i.e., the flux change from the pre-dropout time to the minimum dropout time). (c) The percentage contributed by the magnetopause shadowing (MS) plus the radial diffusion (RD) in the relative flux change.

(67 superposed HSS events from 2005 to 2008) with an explicit time-dependent, $K$-specific magnetopause boundary (i.e., last closed drift shell) in the radial diffusion model to capture the real loss out of the boundary. This study has made another significant further step in quantifying the relative contribution of the effect of magnetopause shadowing coupled with outward radial transport by comparing simulation results with the relative flux change observed by the GPS spacecraft. While previous studies only qualitatively suggested that the above combined effect could mainly explain the electron radiation belt dropouts without showing any percentage contribution to the observed dropout, this work is able to determine the percentage of its contribution. A contribution of $93-99 \%$ is found, suggesting that the above coupled loss mechanism can be primarily responsible for the total electron loss near the geosynchronous position $\left(L^{*}>5\right)$. Nevertheless, some additional loss mechanisms are still needed to fully explain the electron loss in the inner region $\left(L^{*} \leq 5\right)$. This finding based on the numeric percentage is consistent with the qualitative conclusion from previous studies. Note that the diffusion coefficient $D_{\mathrm{LL}}$ used in the 1-D radial diffusion model after Brautigam and Albert (2000) merely represents the diffusion contributed from magnetic field perturbations; however, studies have shown that the contribution from electric field perturbations can be significant (e.g., Ozeke et al., 2012; Tu et al., 2012). But the relative importance between the two is still under study. Therefore, we carried out sensitivity tests by decreasing/increasing the diffusion coefficient $D_{\mathrm{LL}}$ by a factor of 2,5 and 10 and found that a larger diffusion coefficient results in slightly more loss through the magnetopause boundary during the storm main phase and hence the relative contribution by the magnetopause shadowing coupled with the radial diffusion increases by a few percent. This indicates that our quantitative study is embedded with some uncertainty; however this does not change the conclusion that the magnetopause shadowing coupled with the outward radial diffusion is nearly responsible for the radiation belt electron dropout near the geosynchronous orbit.

\section{Conclusions}

Since the fundamental question regarding the primary mechanism responsible for the energetic electron flux depletion in the outer radiation belt remains controversial, this study has made significant steps toward quantifying, rather than qualitatively determining, the effect of magnetopause shadowing on the energetic electron dropouts during 67 HSS events by comparing with GPS dropout observations. Unlike previous radial diffusion simulations with a fixed boundary location, this study utilized a time-varying, $K$-dependent boundary to represent the magnetopause boundary, i.e., the last closed drift shell. Such a boundary setting allows for the explicit identification of flux loss due to magnetopause shadowing. Results indicate that during the small but representative storm events, the drift loss to the magnetopause (i.e, magnetopause shadowing) together with outward radial diffusion is mainly responsible for the electron loss, contributing approximately $93-99 \%$ of the total loss near the geosynchronous orbit $\left(L^{*}>5.0\right)$, but with the inner region $\left(L^{*} \leq 5.0\right)$ requiring some additional loss mechanisms (only $60 \%$ can be explained by the above coupled mechanism).

Future studies will be directed to quantify relative contributions of other individual or mixed loss mechanisms, such as pitch angle and energy diffusions, which can be included within multidimensional models to represent wave-particle interactions (e.g., Beutier and Boscher, 1995; Albert et al., 2009; Su et al., 2010; Subbotin et al., 2010; Tu et al., 2013).

Acknowledgements. This work was conducted under the auspices of the US Department of Energy, with support from the Los Alamos National Laboratory Directed Research and Development program. The authors would like to acknowledge Yue Chen for providing the phase space density data for conducting the DREAM data assimilation in order to obtain the initial conditions used in this study.

Topical Editor L. Blomberg thanks S. Elkington and one anonymous referee for their help in evaluating this paper. 


\section{References}

Albert, J. M., Meredith, N. P., and Horne, R. B.: Three-dimensional diffusion simulation of outer radiation belt electrons during the 9 October 1990 magnetic storm, J. Geophys. Res.-Space, 114, A09214, doi:10.1029/2009JA014336, 2009.

Beutier, T. and Boscher, D.: A three-dimensional analysis of the electron radiation belt by the Salammbô code, J. Geophys. Res., 100, 14853-14862, doi:10.1029/94JA03066, 1995.

Bortnik, J., Thorne, R. M., O’Brien, T. P., Green, J. C., Strangeway, R. J., Shprits, Y. Y., and Baker, D. N.: Observation of two distinct, rapid loss mechanisms during the 20 November 2003 radiation belt dropout event, J. Geophys. Res.-Space, 111, A12216, doi:10.1029/2006JA011802, 2006.

Brautigam, D. H. and Albert, J. M.: Radial diffusion analysis of outer radiation belt electrons during the October 9, 1990, magnetic storm, J. Geophys. Res.-Space, 105, 291-310, doi:10.1029/1999JA900344, 2000.

Cayton, T. E., Belian, R. D., Gary, S. P., Fritz, T. A., and Baker, D. N.: Energetic electron components at geosynchronous orbit, Geophys. Res. Lett., 16, 147-150, doi:10.1029/GL016i002p00147, 1989.

Chen, Y., Friedel, R. H. W., Reeves, G. D., Onsager, T. G., and Thomsen, M. F.: Multisatellite determination of the relativistic electron phase space density at geosynchronous orbit: Methodology and results during geomagnetically quiet times, J. Geophys. Res.-Space, 110, A10210, doi:10.1029/2004JA010895, 2005.

Denton, M. H. and Cayton, T. E.: Density and temperature of energetic electrons in the Earth's magnetotail derived from highlatitude GPS observations during the declining phase of the solar cycle, Ann. Geophys., 29, 1755-1763, doi:10.5194/angeo-291755-2011, 2011.

Denton, M. H., Borovsky, J. E., and Cayton, T. E.: A densitytemperature description of the outer electron radiation belt during geomagnetic storms, J. Geophys. Res.-Space, 115, A01208, doi:10.1029/2009JA014183, 2010.

Desorgher, L., Bühler, P., Zehnder, A., and Flückiger, E. O.: Simulation of the outer radiation belt electron flux decrease during the March 26, 1995, magnetic storm, J. Geophys. Res.-Space, 105, 21211-21224, doi:10.1029/2000JA900060, 2000.

Distel, J. R., Blair, S. G., Cayton, T. E., Dingler, R. D., Guyker, F., Ingraham, J. C., Noveroske, E., Reedy, R. C., Spencer, K. M., and Wehne, T. J.: The Combined X-ray Dosimeter (CXD) on GPS Block IIR Satellites, Tech. Rep. LA-UR-99-2280, Los Alamos National Laboratory, Los Alamos, USA, 1999.

Ginet, G., O’Brien, T., Huston, S., Johnston, W., Guild, T., Friedel, R., Lindstrom, C., Roth, C., Whelan, P., Quinn, R., Madden, D., Morley, S., and Su, Y.-J.: AE9, AP9 and SPM: New Models for Specifying the Trapped Energetic Particle and Space Plasma Environment, Space Sci. Rev., 179, 1-37, doi:10.1007/s11214-0139964-y, 2013.

Hendry, A. T., Rodger, C. J., Clilverd, M. A., Thomson, N. R., Morley, S. K., and Raita, T.: Rapid Radiation Belt Losses Occurring During High-Speed Solar Wind Stream-Driven Storms: Importance of Energetic Electron Precipitation, American Geophysical Union, doi:10.1029/2012GM001299, 213-224, 2012.

Kim, H.-J. and Chan, A. A.: Fully adiabatic changes in storm time relativistic electron fluxes, J. Geophys. Res.-Space, 1022, 22107-22116, doi:10.1029/97JA01814, 1997.
Kim, K. C., Lee, D.-Y., Kim, H.-J., Lyons, L. R., Lee, E. S., Öztürk, M. K., and Choi, C. R.: Numerical calculations of relativistic electron drift loss effect, Journal of Geophysical Research (Space Physics), 113, A09212, doi:10.1029/2007JA013011, 2008.

Kim, K. C., Lee, D.-Y., Kim, H.-J., Lee, E. S., and Choi, C. R.: Numerical estimates of drift loss and Dst effect for outer radiation belt relativistic electrons with arbitrary pitch angle, J. Geophys. Res.-Space, 115, A03208, doi:10.1029/2009JA014523, 2010.

Kim, K. C., Shprits, Y., Subbotin, D., and Ni, B.: Relativistic radiation belt electron responses to GEM magnetic storms: Comparison of CRRES observations with 3-D VERB simulations, J. Gephys. Res., 117, doi:10.1029/2011JA017460, 2012.

Koller, J. and Zaharia, S.: LANL*V2.0: global modeling and validation, Geosci. Model Dev., 4, 669-675, doi:10.5194/gmd-4-6692011, 2011.

Koller, J., Chen, Y., Reeves, G. D., Friedel, R. H. W., Cayton, T. E., and Vrugt, J. A.: Identifying the radiation belt source region by data assimilation, J. Geophys. Res.-Space, 112, A06244, doi:10.1029/2006JA012196, 2007.

Koller, J., Reeves, G. D., and Friedel, R. H. W.: LANL* V1.0: a radiation belt drift shell model suitable for real-time and reanalysis applications, Geosci. Model Dev., 2, 113-122, doi:10.5194/gmd2-113-2009, 2009.

Li, L. Y., Cao, J. B., Zhou, G. C., and Li, X.: Statistical roles of storms and substorms in changing the entire outer zone relativistic electron population, J. Geophys. Res.-Space, 114, A12214, doi:10.1029/2009JA014333, 2009.

Loewe, C. A. and Prölss, G. W.: Classification and mean behavior of magnetic storms, J. Geophys. Res.-Space, 102, 14209-14214, doi:10.1029/96JA04020, 1997.

Loto'aniu, T. M., Singer, H. J., Waters, C. L., Angelopoulos, V., Mann, I. R., Elkington, S. R., and Bonnell, J. W.: Relativistic electron loss due to ultralow frequency waves and enhanced outward radial diffusion, J. Geophys. Res.-Space, 115, A12245, doi:10.1029/2010JA015755, 2010.

Lyons, L. R., Thorne, R. M., and Kennel, C. F.: Pitchangle diffusion of radiation belt electrons within the plasmasphere, J. Geophys. Res.-Space, 77, 3455-3474, doi:10.1029/JA077i019p03455, 1972.

Matsumura, C., Miyoshi, Y., Seki, K., Saito, S., Angelopoulos, V., and Koller, J.: Outer radiation belt boundary location relative to the magnetopause: Implications for magnetopause shadowing, J. Geophys. Res.-Space, 116, A06212, doi:10.1029/2011JA016575, 2011.

Meredith, N. P., Horne, R. B., Lam, M. M., Denton, M. H., Borovsky, J. E., and Green, J. C.: Energetic electron precipitation during high-speed solar wind stream driven storms, J. Geophys. Res.-Space, 116, A05223, doi:10.1029/2010JA016293, 2011.

Millan, R. M., Lin, R. P., Smith, D. M., and McCarthy, M. P.: Observation of relativistic electron precipitation during a rapid decrease of trapped relativistic electron flux, Geopys. Res. Lett., 34, L10101, doi:10.1029/2006GL028653, 2007.

Millan, R. M., Yando, K. B., Green, J. C., and Ukhorskiy, A. Y.: Spatial distribution of relativistic electron precipitation during a radiation belt depletion event, Geophys. Res. Lett., 37, L20103, doi:10.1029/2010GL044919, 2010. 
Miyoshi, Y., Morioka, A., Misawa, H., Obara, T., Nagai, T., and Kasahara, Y.: Rebuilding process of the outer radiation belt during the 3 November 1993 magnetic storm: NOAA and Exos-D observations, J. Geophys. Res.-Space, 108, 1004, doi:10.1029/2001JA007542, 2003.

Morley, S. K., Friedel, R. H., Spanswick, E. L., Reeves, G. D., Steinberg, J. T., Koller, J., Cayton, T., and Noveroske, E.: Dropouts of the outer electron radiation belt in response to solar wind stream interfaces: Global posistioning system observations, P. Roy. Soc., 466, 3329-3350, doi:10.1098/rspa.2010.0078, 2010a.

Morley, S. K., Friedel, R. H. W., Cayton, T. E., and Noveroske, E.: A rapid, global and prolonged electron radiation belt dropout observed with the Global Positioning System constellation, Geophys. Res. Lett., 37, L06102, doi:10.1029/2010GL042772, 2010b.

Morley, S. K., Welling, D. T., Koller, J., Larsen, B. A., Henderson, M. G., and Niehof, J.: SpacePy - A Python-based Library of Tools for the Space Sciences, in: Proceedings of the 9th Python in Science Conference, edited by: van der Walt, S. and Millman, J., 39-45, 2010c.

Ohtani, S., Miyoshi, Y., Singer, H. J., and Weygand, J. M.: On the loss of relativistic electrons at geosynchronous altitude: Its dependence on magnetic configurations and external conditions, J. Geophys. Res.-Space, 114, A01202, doi:10.1029/2008JA013391, 2009.

Ozeke, L. G., Mann, I. R., Murphy, K. R., Rae, I. J., Milling, D. K., Elkington, S. R., Chan, A. A., and Singer, H. J.: ULF wave derived radiation belt radial diffusion coefficients, J. Geophys. Res.-Space, 117, A04222, doi:10.1029/2011JA017463, 2012.

Öztürk, M. K. and Wolf, R. A.: Bifurcation of drift shells near the dayside magnetopause, J. Geophys. Res.-Space, 112, A07207, doi:10.1029/2006JA012102, 2007.

Reeves, G. D., McAdams, K. L., Friedel, R. H. W., and O'Brien, T. P.: Acceleration and loss of relativistic electrons during geomagnetic storms, Geophys. Res. Lett., 30, 1529, doi:10.1029/2002GL016513, 2003.

Reeves, G. D., Chen, Y., Cunningham, G. S., Friedel, R. W. H., Henderson, M. G., Jordanova, V. K., Koller, J., Morley, S. K., Thomsen, M. F., and Zaharia, S.: Dynamic Radiation Environment Assimilation Model: DREAM, Space Weather, 10, S03006, doi:10.1029/2011SW000729, 2012.

Roederer, J. G.: Dynamics of geomagnetically trapped radiation, in: Physics and Chemistry in Space, Springer, Berlin, 1970.

Schulz, M. and Lanzerotti, L. J.: Particle diffusion in the radiation belts, Springer, 1974.

Shabansky, V. P.: Some Processes in the Magnetosphere, Space Sci. Rev., 12, 299-418, doi:10.1007/BF00165511, 1971.

Shprits, Y. Y., Thorne, R. M., Friedel, R., Reeves, G. D., Fennell, J., Baker, D. N., and Kanekal, S. G.: Outward radial diffusion driven by losses at magnetopause, J. Geophys. Res.-Space, 111, A11214, doi:10.1029/2006JA011657, 2006.

Su, Z., Xiao, F., Zheng, H., and Wang, S.: STEERB: A three-dimensional code for storm-time evolution of electron radiation belt, J. Geophys. Res.-Space, 115, A09208, doi:10.1029/2009JA015210, 2010.
Su, Z., Xiao, F., Zheng, H., and Wang, S.: Radiation belt electron dynamics driven by adiabatic transport, radial diffusion, and wave-particle interactions, J. Geophys. Res.-Space, 116, A04205, doi:10.1029/2010JA016228, 2011a.

Su, Z., Xiao, F., Zheng, H., and Wang, S.: CRRES observation and STEERB simulation of the 9 October 1990 electron radiation belt dropout event, Geophys. Res. Lett., 38, L06106, doi:10.1029/2011GL046873, 2011b.

Subbotin, D., Shprits, Y., and Ni, B.: Three-dimensional VERB radiation belt simulations including mixed diffusion, J. Geophys. Res.-Space, 115, A03205, doi:10.1029/2009JA015070, 2010.

Subbotin, D. A., Shprits, Y. Y., and Ni, B.: Long-term radiation belt simulation with the VERB 3-D code: Comparison with CRRES observations, J. Geophys. Res.-Space, 116, A12210, doi:10.1029/2011JA017019, 2011.

Summers, D., Ni, B., and Meredith, N. P.: Timescales for radiation belt electron acceleration and loss due to resonant wave-particle interactions: 1. Theory, J. Geophys. Res.-Space, 112, A04206, doi:10.1029/2006JA011801, 2007a.

Summers, D., Ni, B., and Meredith, N. P.: Timescales for radiation belt electron acceleration and loss due to resonant wave-particle interactions: 2. Evaluation for VLF chorus, ELF hiss, and electromagnetic ion cyclotron waves, J. Geophys. Res.-Space, 112, A04207, doi:10.1029/2006JA011993, 2007b.

Thorne, R. M., O'Brien, T. P., Shprits, Y. Y., Summers, D., and Horne, R. B.: Timescale for MeV electron microburst loss during geomagnetic storms, J. Geophys. Res.-Space, 110, A09202, doi:10.1029/2004JA010882, 2005.

Tsyganenko, N. A.: A magnetospheric magnetic field model with a warped tail current sheet, Planet. Space Sci., 37, 5-20, doi:10.1016/0032-0633(89)90066-4, 1989.

Tu, W., Elkington, S. R., Li, X., Liu, W., and Bonnell, J.: Quantifying radial diffusion coefficients of radiation belt electrons based on global MHD simulation and spacecraft measurements, J. Geophys. Res.-Space, 117, A10210, doi:10.1029/2012JA017901, 2012.

Tu, W., Cunningham, G. S., Chen, Y., Henderson, M. G., Camporeale, E., and Reeves, G. D.: Modeling radiation belt electron dynamics during GEM challenge intervals with the DREAM3D diffusion model, J. Geophys. Res.-Space, doi:10.1002/jgra.50560, in press, 2013.

Turner, D. L., Shprits, Y., Hartinger, M., and Angelopoulos, V.: Explaining sudden losses of outer radiation belt electrons during geomagnetic storms, Nat. Phys., 8, 208-212, doi:10.1038/nphys2185, 2012.

Turner, D. L., Morley, S. K., Miyoshi, Y., Ni, B., and Huang, C. L.: Outer radiation belt flux dropouts: Current understanding and unresolved questions, Washington DC American Geophysical Union Geophysical Monograph Series, 199, 195-212, 2013.

Ukhorskiy, A. Y., Anderson, B. J., Brandt, P. C., and Tsyganenko, N. A.: Storm time evolution of the outer radiation belt: Transport and losses, J. Geophys. Res.-Space, 111, A11S03, doi:10.1029/2006JA011690, 2006.

Ukhorskiy, A. Y., Sitnov, M. I., Millan, R. M., and Kress, B. T.: The role of drift orbit bifurcations in energization and loss of electrons in the outer radiation belt, J. Geophys. Res.-Space, 116, A09208, doi:10.1029/2011JA016623, 2011. 
Varotsou, A., Friedel, R. H., Reeves, G. D., Lavraud, B., Skoug, R. M., Cayton, T. E., and Bourdarie, S.: Characterization of relativistic electron flux rise times during the recovery phase of geomagnetic storms as measured by the NS41 GPS satellite, J. Atmos. Sol.-Terr. Phy., 70, 1745-1759, doi:10.1016/j.jastp.2008.01.020, 2008.
Yu, Y., Koller, J., Zaharia, S., and Jordanova, V.: L* neural networks from different magnetic field models and their applicability, Space Weather, 10, S02014, doi:10.1029/2011SW000743, 2012. 